\title{
A FOCAL SUBGROUP THEOREM FOR OUTER COMMUTATOR WORDS
}

\author{
CRISTINA ACCIARRI, GUSTAVO A. FERNÁNDEZ-ALCOBER, \\ AND PAVEL SHUMYATSKY
}

\begin{abstract}
Let $G$ be a finite group of order $p^{a} m$, where $p$ is a prime and $m$ is not divisible by $p$, and let $P$ be a Sylow $p$-subgroup of $G$. If $w$ is an outer commutator word, we prove that $P \cap w(G)$ is generated by the intersection of $P$ with the set of $m$ th powers of all values of $w$ in $G$.
\end{abstract}

Let $G$ be a finite group and $P$ a Sylow $p$-subgroup of $G$. The Focal Subgroup Theorem states that $P \cap G^{\prime}$ is generated by the set of commutators $\{[g, z] \mid g \in G, z \in P,[g, z] \in P\}$. This was proved by Higman in $1953[5]$. Nowadays the proof of the theorem can be found in many standard books on group theory (for example, Rose's book [7] or Gorenstein's [3]).

One immediate corollary is that $P \cap G^{\prime}$ can be generated by commutators lying in $P$. Of course, $G^{\prime}$ is the verbal subgroup of $G$ corresponding to the group word $[x, y]=x^{-1} y^{-1} x y$. It is natural to ask the question on generation of Sylow subgroups for other words. More specifically, if $w$ is a group word we write $G_{w}$ for the set of values of $w$ in $G$ and $w(G)$ for the subgroup generated by $G_{w}$ (which is called the verbal subgroup of $w$ in $G$ ), and one is tempted to ask the following question.

Question. Given a Sylow p-subgroup $P$ of a finite group $G$, is it true that $P \cap w(G)$ can be generated by $w$-values lying in $P$, i.e., that $P \cap w(G)=$ $\left\langle P \cap G_{w}\right\rangle$ ?

However considering the case where $G$ is the non-abelian group of order six, $w=x^{3}$ and $p=3$ we quickly see that the answer to the above question is negative. Therefore we concentrate on the case where $w$ is a commutator word. Recall that a group word is commutator if the sum of the exponents of any indeterminate involved in it is zero. Thus, we deal with the question whether $P \cap w(G)$ can be generated by $w$-values whenever $w$ is a commutator word.

The main result of this paper is a contribution towards a positive answer to this question: we prove that if $w$ is an outer commutator word, then $P \cap w(G)$ can be generated by the powers of values of $w$ which lie in $P$. More precisely, we have the following result.

Theorem A. Let $G$ be a finite group of order $p^{a} m$, where $p$ is a prime and $m$ is not divisible by $p$, and let $P$ be a Sylow p-subgroup of $G$. If $w$ is

2010 Mathematics Subject Classification. Primary 20D20; secondary 20 F12.

Key words and phrases. Sylow subgroups, verbal subgroups, focal subgroups, outer commutator words.

The second author is supported by the Spanish Government, grant MTM2008-06680C02-02, partly with FEDER funds, and by the Basque Government, grant IT-460-10. The third author is supported by CNPq-Brazil. 
an outer commutator word, then $P \cap w(G)$ is generated by mth powers of $w$-values, i.e., $P \cap w(G)=\left\langle P \cap G_{w^{m}}\right\rangle$.

Recall that an outer commutator word is a word which is obtained by nesting commutators, but using always different indeterminates. Thus the word $\left[\left[x_{1}, x_{2}\right],\left[x_{3}, x_{4}, x_{5}\right], x_{6}\right]$ is an outer commutator while the Engel word $\left[x_{1}, x_{2}, x_{2}, x_{2}\right]$ is not. An important family of outer commutator words are the simple commutators $\gamma_{i}$, given by

$$
\gamma_{1}=x_{1}, \quad \gamma_{i}=\left[\gamma_{i-1}, x_{i}\right]=\left[x_{1}, \ldots, x_{i}\right], \quad \text { for } i \geq 2 .
$$

The corresponding verbal subgroups $\gamma_{i}(G)$ are the terms of the lower central series of $G$. Another distinguished sequence of outer commutator words are the derived words $\delta_{i}$, on $2^{i}$ indeterminates, which are defined recursively by

$$
\delta_{0}=x_{1}, \quad \delta_{i}=\left[\delta_{i-1}\left(x_{1}, \ldots, x_{2^{i-1}}\right), \delta_{i-1}\left(x_{2^{i-1}+1}, \ldots, x_{2^{i}}\right)\right], \quad \text { for } i \geq 1 .
$$

Then $\delta_{i}(G)=G^{(i)}$, the $i$ th derived subgroup of $G$.

Some of the ideas behind the proof of Theorem A were anticipated already in 4 where somewhat similar arguments, due to Guralnick, led to a result on generation of a Sylow $p$-subgroup of $G^{\prime}$ for a finite group $G$ admitting a coprime group of automorphisms. Later the arguments were refined in [1]. In both papers [4] and [1] the results on generation of Sylow subgroups were used to reduce a problem about finite groups to the case of nilpotent groups. It is hoped that also our Theorem A will play a similar role in the subsequent projects.

Another important tool used in the proof of Theorem A is the famous result of Liebeck, O'Brien, Shalev and Tiep [6] that every element of a nonabelian simple group is a commutator. The result proved Ore's conjecture thus solving a long-standing problem. In turn, the proof in [6] uses the classification of finite simple groups as well as many other sophisticated tools.

\section{Preliminaries}

If $X$ and $Y$ are two subsets of a group $G$, and $N$ is a normal subgroup of $G$, it is not always the case that $X N \cap Y N=(X \cap Y) N$, i.e., that $\bar{X} \cap \bar{Y}=\overline{X \cap Y}$ in the quotient group $\bar{G}=G / N$. In our first lemma we have a situation in which this property holds, and which will be of importance in the sequel.

Lemma 1.1. Let $G$ be a finite group, and let $N$ be a normal subgroup of $G$. If $P$ is a Sylow p-subgroup of $G$ and $X$ is a normal subset of $G$ consisting of p-elements, then $X N \cap P N=(X \cap P) N$. In other words, if we use the bar notation in $G / N$, we have $\bar{X} \cap \bar{P}=\overline{X \cap P}$.

Proof. We only need to worry about the inclusion $\bar{X} \cap \bar{P} \subseteq \overline{X \cap P}$. So, given an element $g \in X N \cap P N$, we prove that $g \in x N$ for some $x \in X \cap P$. Since we have $g \in X N$, we may assume without loss of generality that $g \in X$, and in particular $g$ is a $p$-element. Since also $g \in P N$, there exists $z \in P$ such that $g N=z N$.

Put $H=\langle g\rangle N=\langle z\rangle N$, and observe that $H^{\prime} \leq N$. Since $P \cap N$ is a Sylow $p$-subgroup of $N$ and $z \in P$, it follows that $P \cap H=\langle z\rangle(P \cap N)$ is a Sylow $p$-subgroup of $H$. Now, $g$ is a $p$-element of $H$, and so we get $g^{h} \in P \cap H$ for 
some $h \in H$. If we put $x=g^{h}$ then $x \in X \cap P$, since $X$ is a normal subset of $G$, and $g=x^{h^{-1}}=x\left[x, h^{-1}\right] \in x H^{\prime} \subseteq x N$, as desired.

The next lemma will be fundamental in the proof of Theorem A, since it will allow us to go up a series from 1 to $w(G)$ in which all quotients of two consecutive terms are verbal subgroups of a word all of whose values are also $w$-values.

Lemma 1.2. Let $G$ be a finite group, and let $P$ be a Sylow p-subgroup of $G$. Assume that $N \leq L$ are two normal subgroups of $G$, and use the bar notation in the quotient group $G / N$. Let $X$ be a normal subset of $G$ consisting of p-elements such that $\bar{P} \cap \bar{L}=\langle\bar{P} \cap \bar{X}\rangle$. Then $P \cap L=\langle P \cap X, P \cap N\rangle$.

Proof. By Lemma 1.1, we have $\bar{P} \cap \bar{L}=\langle\overline{P \cap X}\rangle$, and this implies that $P N \cap L=\langle P \cap X\rangle N$. By intersecting with $P$, we get

$$
P \cap L=P \cap(P N \cap L)=P \cap\langle P \cap X\rangle N=\langle P \cap X\rangle(P \cap N),
$$

where the last equality follows from Dedekind's law. This proves the result.

We will also need the following lemma, of a different nature.

Lemma 1.3. Let $G$ be a finite group, and let $N$ be a minimal normal subgroup of $G$. If $N$ does not contain any non-trivial elements of $G_{\delta_{i}}$, where $i \geq 1$, then $\left[N, G^{(i-1)}\right]=1$.

Proof. We argue by induction on $i$. If $i=1$ then, since $N$ is normal in $G$ and does not contain any non-trivial commutators of elements of $G$, it follows that $[n, g]=1$ for every $n \in N$ and $g \in G$. Thus $[N, G]=1$, as desired.

Assume now that $i \geq 2$. The fact that $N$ is a minimal normal subgroup of $G$ implies that the subgroup $\left\langle N \cap G_{\delta_{i-1}}\right\rangle$ must be either equal to $N$ or the trivial subgroup. In the former case, we have $N=\left\langle N \cap G_{\delta_{i-1}}\right\rangle$ and so $\left[N, G^{(i-1)}\right]$ is generated by elements of the form $[a, b]$ where $a \in N \cap G_{\delta_{i-1}}$ and $b \in G_{\delta_{i-1}}$. In particular, each commutator $[a, b]$ belongs to $N \cap G_{\delta_{i}}$ and must be 1 by the hypothesis. Hence $\left[N, G^{(i-1)}\right]=1$. If $N \cap G_{\delta_{i-1}}=1$, then it follows from the induction hypothesis that $\left[N, G^{(i-2)}\right]=1$, and the result holds.

We conclude this preliminary section by showing that Theorem A holds for every word under the assumption that the verbal subgroup $w(G)$ is nilpotent.

Theorem 1.4. Let $G$ be a finite group of order $p^{a} m$, where $p$ is a prime and $m$ is not divisible by $p$, and let $P$ be a Sylow p-subgroup of $G$. If $w$ is any word such that $w(G)$ is nilpotent, then

$$
P \cap w(G)=\left\langle P \cap G_{w^{m}}\right\rangle .
$$

Proof. By Bezout's identity, there exist two integers $\lambda$ and $\mu$ such that $1=\lambda p^{a}+\mu m$. If we put $u=w^{p^{a}}$ and $v=w^{m}$, then for every $g \in G_{w}$ we have

$$
g=\left(g^{p^{a}}\right)^{\lambda} \cdot\left(g^{m}\right)^{\mu} \in\left\langle G_{u}\right\rangle \cdot\left\langle G_{v}\right\rangle .
$$


Hence

$$
w(G)=\left\langle G_{u}, G_{v}\right\rangle
$$

Note that all elements of $G_{u}$ have $p^{\prime}$-order, and all elements of $G_{v}$ have $p$ power order. Since $w(G)$ is nilpotent, it follows that $\left\langle G_{u}\right\rangle$ is a $p^{\prime}$-subgroup of $w(G),\left\langle G_{v}\right\rangle$ is a $p$-subgroup, and $G_{u}$ and $G_{v}$ commute elementwise. As a consequence of this and (11), we get

$$
w(G)=\left\langle G_{u}\right\rangle \times\left\langle G_{v}\right\rangle,
$$

and $\left\langle G_{u}\right\rangle$ and $\left\langle G_{v}\right\rangle$ are a Hall $p^{\prime}$-subgroup and a Sylow $p$-subgroup of $w(G)$, respectively. We conclude that $P \cap w(G)=\left\langle G_{v}\right\rangle$, which proves the theorem.

\section{The proof of Theorem A}

The first step in the proof of Theorem $\mathrm{A}$ is to verify it for $\delta_{i}$, which is done in Theorem 2.3 below. For this, we will rely on the result by Liebeck, O'Brien, Shalev and Tiep 6] that proved Ore's conjecture, according to which every element of a non-abelian simple group is a commutator, and $a$ fortiori, also a value of $\delta_{i}$ for every $i$. We will also need the following result of Gaschütz (see page 191 of [8]).

Theorem 2.1. Let $G$ be a finite group, and let $P$ be a Sylow p-subgroup of $G$. If $N$ is a normal abelian p-subgroup of $G$, then $N$ is complemented in $G$ if and only if $N$ is complemented in $P$.

In the proof of Theorem $\mathrm{A}$ for both $\delta_{i}$ and an arbitrary outer commutator word, we will argue by induction. Then it will be important to take into account the following remark.

Remark 2.2. Let $G$ be a group of order $p^{a} m$ for which we want to prove Theorem $\mathrm{A}$ in the case of a given word $w$. Assume that $K$ is a group whose order $p^{a^{*}} m^{*}$ is a divisor of $p^{a} m$ (for example, a subgroup or a quotient of $G$ ), and let $P^{*}$ be a Sylow $p$-subgroup of $K$. If Theorem $\mathrm{A}$ is known to hold for $K$ and $w$, then we have $P^{*} \cap w(K)=\left\langle P^{*} \cap K_{w^{*}}\right\rangle$. Since $m / m^{*}$ is a positive integer which is coprime to $p$, it follows that $P^{*} \cap w(K)=\left\langle\left(P^{*} \cap K_{w^{m}}\right)^{m / m^{*}}\right\rangle$, and so also that $P^{*} \cap w(K)=\left\langle P^{*} \cap K_{w^{m}}\right\rangle$. In other words, in the statement of Theorem A for $K$, we can replace the power word $w^{m^{*}}$ corresponding to the order of $K$ with the word $w^{m}$, which corresponds to the order of $G$.

We can now proceed to the proof of Theorem A for $\delta_{i}$.

Theorem 2.3. Let $G$ be a finite group of order $p^{a} m$, where $p$ is a prime and $m$ is not divisible by $p$, and let $P$ be a Sylow p-subgroup of $G$. Then, for every $i \geq 0$, we have

$$
P \cap G^{(i)}=\left\langle P \cap G_{\delta_{i}^{m}}\right\rangle .
$$

Proof. We argue by induction on the order of $G$. The result is obvious if either $i=0$ or $G^{(i)}=1$, so we assume that $i \geq 1$ and $G^{(i)} \neq 1$.

Let $N \neq 1$ be a normal subgroup of $G$ which is contained in $G^{(i)}$. Then the result holds in $\bar{G}=G / N$, and we have $\bar{P} \cap \bar{G}^{(i)}=\left\langle\bar{P} \cap \bar{G}_{\delta_{i}^{m}}\right\rangle$. By applying Lemma 1.2, we get

$$
P \cap G^{(i)}=\left\langle P \cap G_{\delta_{i}^{m}}, P \cap N\right\rangle .
$$


Now we assume that $N$ is a minimal normal subgroup of $G$, and we consider three different cases, depending on the structure of $N$.

(i) $N$ is a direct product of isomorphic non-abelian simple groups.

By the positive solution to Ore's conjecture, we have $N=N_{\delta_{i}}$. Hence we get $P \cap N \subseteq N_{\delta_{i}}$, and since the map $z \mapsto z^{m}$ is a bijection in $P \cap N$, it follows that $P \cap N \subseteq P \cap N_{\delta_{i}^{m}}$. Now the result is immediate from (3).

(ii) $N \cong C_{q} \times \cdots \times C_{q}$, where $q$ is a prime different from $p$.

In this case, $P \cap N=1$ and the result obviously holds.

(iii) $N \cong C_{p} \times \cdots \times C_{p}$.

In this case, we have $N \leq P$ and so $P \cap N=N$. Since $\left\langle N \cap G_{\delta_{i}}\right\rangle$ is a normal subgroup of $G$ and $N$ is a minimal normal subgroup, it follows that either $\left\langle N \cap G_{\delta_{i}}\right\rangle=N$ or $N \cap G_{\delta_{i}}=1$. In the former case, we have $N=\left\langle\left(N \cap G_{\delta_{i}}\right)^{m}\right\rangle$, since $N$ is a finite $p$-group, and so $N=\left\langle N \cap G_{\delta_{i}^{m}}\right\rangle$ and the theorem follows again from (3). So we are necessarily in the latter case, and then by Lemma 1.3 , we have $\left[N, G^{(i-1)}\right]=1$.

If $G$ is not perfect, then the theorem holds by induction in $G^{\prime}$, and so $P \cap G^{(i+1)}=P \cap\left(G^{\prime}\right)^{(i)}$ can be generated by values of $\delta_{i}^{m}$ lying in $P$. If $G^{(i+1)} \neq 1$ then we can use (3) with $G^{(i+1)}$ in the place of $N$, and we are done. On the other hand, if $G^{(i+1)}=1$ then $G^{(i)}$ is abelian, and the result is a consequence of Theorem 1.4.

Thus we may assume that $G$ is perfect. Then $P \cap G^{(i)}=P$. Also $[N, G]=$ $\left[N, G^{(i-1)}\right]=1$, and $N$ is central in $G$. Being a minimal normal subgroup of $G$, this implies that $|N|=p$. If $N \leq \Phi(P)$ then it follows from (3) that $P=\left\langle P \cap G_{\delta_{i}^{m}}\right\rangle$, as desired. Hence we may assume that $N$ is not contained in a maximal subgroup $M$ of $P$. Since $|N|=p$, it follows that $M$ is a complement of $N$ in $P$. By Theorem 2.1, it follows that $N$ has also a complement in $G$, say $H$. Since $N \leq Z(G)$, we conclude that $G=H \times N$, a contradiction with the fact that $G$ is perfect. This completes the proof.

We will deal with arbitrary outer commutator words using some concepts from the paper [2, where outer commutator words are represented by binary rooted trees in the following way: indeterminates are represented by an isolated vertex, and if $w=[u, v]$ is the commutator of two outer commutator words $u$ and $v$, then the tree $T_{w}$ of $w$ is obtained by drawing the trees $T_{u}$ and $T_{v}$, and a new vertex (which will be the root of the new tree) which is then connected to the roots of $T_{u}$ and $T_{v}$. For example, the following are the trees for the words $\gamma_{4}$ and $\delta_{3}$ (we also label every vertex with the outer commutator word which is represented by the tree appearing on top of that vertex):
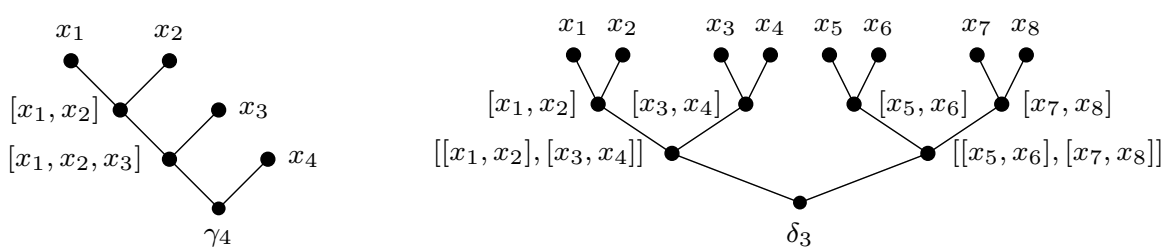

Figure 1. The trees of the words $\gamma_{4}$ and $\delta_{3}$. 
Each of these trees has a visual height, which coincides with the largest distance from the root to another vertex of the tree. Observe that the full binary tree of height $i$ corresponds to the derived word $\delta_{i}$. The following two concepts, also introduced in [2], will be essential in our proof of Theorem A.

Definition 2.4. Let $u$ and $w$ be two outer commutator words. We say that $u$ is an extension of $w$ if the tree of $u$ is an upward extension of the tree of $w$. If $u \neq w$, we say that $u$ is a proper extension of $w$.

An important remark is that, if $u$ is an extension of $w$, then $G_{u} \subseteq G_{w}$.

Definition 2.5. If $w$ is an outer commutator word whose tree has height $i$, the defect of $w$ is the number of vertices that need to be added to the tree of $w$ in order to get the tree of $\delta_{i}$. In other words, if the tree of $w$ has $V$ vertices, the defect of $w$ is $2^{i+1}-1-V$.

Thus the only words of defect 0 are the derived words. Our proof of Theorem A also depends on the following result, which is implicit in the proof of Theorem B of [2].

Theorem 2.6. Let $w=[u, v]$ be an outer commutator word of height $i$, different from $\delta_{i}$. Then at least one of the subgroups $[w(G), u(G)]$ and $[w(G), v(G)]$ is contained in a product of verbal subgroups corresponding to words which are proper extensions of $w$ of height $i$.

Let us now give the proof of Theorem A.

Proof of Theorem A. We argue by induction on the defect of the word $w$. If the defect is 0 , then $w$ is a derived word, and the result is true by Theorem 2.3. Hence we may assume that the defect is positive. If the height of $w$ is $i$, let $\Phi=\left\{\varphi_{1}, \ldots, \varphi_{r}\right\}$ be the set of all outer commutator words of height $i$ which are a proper extension of $w$. Since every word in the set $\Phi$ has smaller defect than $w$, the theorem holds for all $\varphi_{i}$.

Put $N_{0}=1, N_{i}=\varphi_{1}(G) \ldots \varphi_{i}(G)$ for $1 \leq i \leq r$, and $N=N_{r}$. Let us write $w=[u, v]$, where $u$ and $v$ are outer commutator words. Since $[w(G), w(G)]$ is contained in both $[w(G), u(G)]$ and $[w(G), v(G)]$, it follows from Theorem [2.6 that $[w(G), w(G)] \leq N$. Thus if $\bar{G}=G / N$, the verbal subgroup $w(\bar{G})$ is abelian, and so Theorem A holds in $\bar{G}$, according to Theorem [1.4. Hence $\bar{P} \cap w(\bar{G})=\left\langle\bar{P} \cap \bar{G}_{w^{m}}\right\rangle$, and by applying Lemma 1.2, we get $P \cap w(G)=\left\langle P \cap G_{w^{m}}, P \cap N\right\rangle$.

Consequently, it suffices to show that $P \cap N$ can be generated by values of $w^{m}$. We see this by proving that $P \cap N_{i}=\left\langle P \cap N_{i} \cap G_{w^{m}}\right\rangle$ for every $i=0, \ldots, r$, by induction on $i$. There is nothing to prove if $i=0$, so we assume that $i \geq 1$. If $\bar{G}=G / N_{i-1}$, we have $\bar{N}_{i}=\varphi_{i}(\bar{G})$. Since the theorem is true for $\varphi_{i}$, it follows that $\bar{P} \cap \bar{N}_{i}=\left\langle\bar{P} \cap \bar{G}_{\varphi_{i}^{m}}\right\rangle$. By Lemma 1.2, we get

$$
P \cap N_{i}=\left\langle P \cap G_{\varphi_{i}^{m}}, P \cap N_{i-1}\right\rangle .
$$

Observe that, since $\varphi_{i}$ is an extension of $w$, we have $G_{\varphi_{i}^{m}} \subseteq G_{w^{m}}$. Since also $G_{\varphi_{i}^{m}} \subseteq \varphi_{i}(G) \leq N_{i}$, we can further say that $G_{\varphi_{i}^{m}} \subseteq N_{i} \cap G_{w^{m}}$. Hence

$$
P \cap N_{i}=\left\langle P \cap N_{i} \cap G_{w^{m}}, P \cap N_{i-1}\right\rangle,
$$

and the result follows from the induction hypothesis. 


\section{REFERENCES}

[1] C. Acciarri, P. Shumyatsky, Fixed points of coprime operator groups, J. Algebra 342 (2011), 161-174.

[2] G.A. Fernández-Alcober, M. Morigi, Outer commutator words are uniformly concise, J. London Math. Soc. 82 (2010), 581-595.

[3] D. Gorenstein, Finite Groups, Chelsea Publishing Company, New York, 1980.

[4] R. Guralnick, P. Shumyatsky, Derived subgroups of fixed points, Israel J. Math. 126 (2001), 345-362.

[5] G. Higman, Focal series in finite groups, Canad. J. Math. 5 (1953), 477-497.

[6] M.W. Liebeck, E.A. O'Brien, A. Shalev, P.H. Tiep, The Ore conjecture. J. Eur. Math. Soc., 12(4) (2010), 939-1008.

[7] J.S. Rose, A Course in Group Theory, Dover Publications, New York, 1994.

[8] J.J. Rotman, An Introduction to the Theory of Groups, 4th ed., Springer-Verlag, 1995.

Department of Mathematics, University of Brasilia, Brasilia-DF, 70910-900 BRAZIL

E-mail address: acciarricristina@yahoo.it

Matematika Saila, Euskal Herriko Unibertsitatea, 48080 Bilbao, Spain

E-mail address: gustavo.fernandez@ehu.es

Department of Mathematics, University of Brasilia, Brasilia-DF, 70910-900 BRAZIL

E-mail address: pavel@unb.br 